\begin{tabular}{|l|l|l|l|l|}
\hline $\mathrm{M}$ & $\mathrm{R}$ & $\mathrm{S}$ & Internet Journal of & Nitride Semiconductor Research \\
\hline
\end{tabular}

Volume 2, Article 31

\title{
Comparison of Luminescence and Physical Morphologies of GaN Epilayers
}

\author{
Carol Trager-Cowan, P. G. Middleton, K. P. O'Donnell \\ Department of Physics and Applied Physics, University of Strathclyde \\ S. Ruffenach-Clur, Olivier Briot \\ Groupe d'Etude des Semiconducteurs, GES-CNRS
}

This article was received on June 17, 1997 and accepted on September 15, 1997.

\begin{abstract}
In this paper we examine a series of four GaN epilayers grown by MOVPE on sapphire substrates with different AIN buffer layer thicknesses. We examine the effect of the buffer layer thickness on the physical and optical properties of the samples via optical microscopy, cathodoluminescence imaging and photoluminescence and cathodoluminescence spectroscopy. While the morphological and optical properties of all the films (excepting that with the thinnest buffer layer of $30 \mathrm{~nm}$ ) are good, i.e., the films are smooth and the luminescence is dominated by excitonic luminescence, a number of circular island like features are observed in all the films whose density decrease with increasing buffer layer thickness. A large circular island present on the sample with the thinnest buffer layer and surrounded by cracks in the $<11 \overline{2} \quad 0>$ directions, displays some interesting acceptor related luminescence.
\end{abstract}

\section{Introduction}

The nitrides are at the present time exciting materials to study, not only because of their potential to provide us with the elusive commercial blue laser diode [1], but because they are physically interesting due to their variable and often exceptional morphologies and associated optical properties. This makes the imaging of a sample's physical morphology as important as the acquisition of luminescence spectra.

In previous work [2], [3], [4], [5], [6], [7], we have illustrated the power of coupling morphological measurements with luminescence measurements using scanning electron microscopy (SEM) and coincident cathodoluminescence (CL) imaging. We complement these techniques with optical and atomic force microscopies and CL and photoluminescence $(\mathrm{PL})$ spectroscopies. On examination of samples from many different laboratories we have observed a range of morphologies, from samples comprising closely spaced hexagonal crystallites to samples which appear smooth apart from some incidental circular island features (see present paper). In some cases CL images reveal structure not observed with conventional imaging techniques such as optical microscopy. For example CL reveals the presence of a defect (possibly an inversion domain boundary) in the centre of a hexagonal growth hillock [6] in a GaN epilayer.

In this paper we will describe results obtained from a series of four GaN epilayers grown by MOVPE on sapphire substrates with an intervening AIN buffer layer. We will examine in particular the effect of the buffer layer thickness on the physical and optical properties of the samples.

\section{Experimental details}

\subsection{Growth}

The samples were grown on c-plane sapphire substrates using low-pressure MOVPE (ASM OMR 12 reactor) at 76 Torr. The precursors were triethylgallium (TEGa) and ammonia $\left(\mathrm{NH}_{3}\right)$. After nitriding of the substrates at high temperature $\left(1070^{\circ} \mathrm{C}\right)$ in an ambient ammonia atmosphere, an $\left(800^{\circ} \mathrm{C}\right)$ AIN buffer layer was deposited. The temperature of the sample was then raised to the growth temperature of $980^{\circ} \mathrm{C}$ and the buffer layer allowed to recrystallise for two minutes before the GaN epilayer was deposited. The V/III ratio was 10000 . For a discussion of previous work on the effect of growth parameters such as growth temperature, buffer layer thickness and V/III molar 
ratio on epilayer quality, see [8]. The table below summarises the properties of the samples studied in the present work.

\subsection{Optical Microscopy}

An Olympus $\mathrm{BH} 2$ metallurgical microscope equipped with a CCD camera was used to obtain optical micrographs of the samples. Images were acquired using a frame grabbing card installed in a personal computer.

\subsection{PL Spectroscopy}

An Omnichrome Series $56 \mathrm{He}-\mathrm{Cd}$ laser providing several milliwatts of light at $325 \mathrm{~nm}$ was used to obtain PL spectra. The sample temperature may be varied from 15 to $300 \mathrm{~K}$ using a closed cycle cryorefrigerator.

\subsection{Scanning Electron Microscopy and Cathodoluminescence Imaging}

A scanning electron (SE) microscope has been modified to enable light, i.e., cathodoluminescence (CL), to be detected as the electron beam is scanned over the surface of a sample. The resultant CL micrograph can be compared with an SE micrograph obtained from the same area of the sample, allowing a direct comparison of the optical morphology of a sample with its physical morphology. By inserting appropriate absorption filters or a monochromator into the luminescence exit beam, CL images that correspond to selected wavelengths of emission are readily obtained. SE and CL micrographs are acquired by digitisation of the images by a frame grabbing card installed in a personal computer. Spectra may be acquired from a specific area of the sample by scanning the monochromator.

\subsection{Cathodoluminescence Spectroscopy}

A home-built cathodoluminescence spectrometer provides an electron beam with energies up to $30 \mathrm{keV}$ at current densities to, $20 \mathrm{~A} / \mathrm{cm}^{2}$ ( but limited in the work to be described here to less than $1 \mathrm{~mA} / \mathrm{cm}^{2}$ ). The electron beam excitation spot may be focussed to a region of diameter $200 \mathrm{~mm}$, or defocussed to illuminate a whole sample, up to a diameter of about $5 \mathrm{~mm}$. Spectral acquisition in the range of photon energies from 2.0 to $3.5 \mathrm{eV}$ utilises either a R928 Hamamatsu photomultiplier tube or an Oriel InstaSpecTM cooled 2-dimensional CCD array mounted at the output focal plane of a Chromex 0.5M monochromator. Use of the CCD array brings the following advantages: (1) Entire spectra are obtained very quickly. Spectra presented in this paper were obtained with an acquisition time of 10 seconds. (2) Some limited spatial resolution is achieved within the excited region of the sample. The CCD comprises 1024 by 256 pixels, with each pixel measuring $27 \mathrm{~mm}$ square. The luminescence from the sample is dispersed in the horizontal plane and "imaged" in the vertical plane. A single spectrum may be acquired by integrating the signals over the rows of the CCD. Alternatively $n$-spectra $(1 \leq n \leq 256)$ may be acquired by dividing the array via software into vertical tracks. The spectra presented in this paper were acquired by integrating the signal over all 256 rows of the CCD.

\section{Results and discussion}

\subsection{Optical Microscopy}

Under the optical microscope these four samples exhibit a predominantly smooth morphology. However some circular island like features observed on these samples are illustrated in Figure 1. Note the dark core at the centre of most of the islands. Dark spots are also observed which do not coincide with the island centres. Note also that some of the circular islands are surrounded by dark rings, which may indicate the presence of an indented region (or hollow) around the islands, i.e., some of the islands appear to be surrounded by a moat like feature. The circular islands as illustrated in Figure 1 are found on all 4 samples, with the density of these islands decreasing with increasing thickness of the buffer layer.

On the sample with the thinnest buffer layer (B73), a large island is observed surrounded by cracks in the $\langle 11 \overline{2} \quad 0>$ directions as shown in Figure 2.

\subsection{PL Spectroscopy}

Figures 3 and 4 compare typical low temperature (15 K) PL spectra of all four samples obtained from the front and back faces (through the substrate), respectively. Figure 3 shows that apart from the sample B73 which shows acceptor related luminescence near $3.27 \mathrm{eV}$ [9], [10], the luminescence from the epilayers is predominantly excitonic, indicating that the optical quality of all these samples (with the exception of B73) is good. High resolution scans of the excitonic region from each of the samples reveal the exciton band to comprise three bands. Figure 5 shows high resolution PL spectra acquired as a function of position (spectra acquired $>1 \mathrm{~mm}$ apart) across sample B72 (sample with thickest buffer layer). Note the shift ( $4 \mathrm{meV})$ of the peaks as we move from one end of the sample to the other. The origin of this fine structure and their shift will be the subject of further work. 
By exciting through the substrate (i.e., spectra of Figure 4) which is transparent to $325 \mathrm{~nm}$ radiation, we should be able to observe luminescence dominated by the interface between the AIN buffer layer and epilayer. Figure 4 shows that $\mathrm{PL}$ spectra from this region of the sample contains acceptor related luminescence (at $3.3 \mathrm{eV}$ ) [9], [10] and yellow defect luminescence (peaking at $2.25 \mathrm{eV}$ ) as well as excitonic luminescence at $3.43 \mathrm{eV}$ and $3.48 \mathrm{eV}$ respectively [8], [9], [10]. A comparison of Figures 3 and 4 emphasises how the optical quality of the epilayer changes from the interface of the epilayer with the buffer layer and the surface of the epilayer.

\subsection{Scanning Electron Microscopy, Cathodoluminescence Imaging and Spectroscopy}

Scanning electron microscopy and CL imaging also showed the four samples to have a predominantly smooth surface morphology. In general the films emit excitonic CL uniformly at about $355 \mathrm{~nm}$, when excited by $7.5 \mathrm{keV}$ electrons (Bethe range $370 \mathrm{~nm}$ ), at a temperature of $35 \mathrm{~K}$.

We attempted to obtain CL images of the circular islands revealed by optical microscopy, but found that such images showed little contrast at any wavelength (it was not possible to differentiate between the circular features and the material surrounding them), except at their centres from which no luminescence was observed. However we did find one exception to the rule, Figure 6 shows one circular feature on sample B73 where CL provided contrast between it and the surrounding material. This feature was detected because of its strongly luminescencing core around $355 \mathrm{~nm}$. The CL images of Figure 6 were acquired at a beam energy of $7.5 \mathrm{keV}$ and a temperature of $>35 \mathrm{~K}$ under identical excitation and detection conditions. A possible explanation for emission being observed from the centre of this circular feature is that its core is hollow, and excitonic emission is channelled to the surface. Note the almost completely dark (non luminescing) outer ring in Figure 6 (a), this region corresponds to the brightly luminescencing regions in Figure 6 (b). What we are observing is the existence of regions for which excitonic emission dominates and regions for which the acceptor related emission dominates. The spectrum from sample B73 of Figure 4 shows that acceptor related emission predominates for this sample when the luminescence originates from the epilayer at its interface with buffer layer, this implies that the material giving rise to the bright areas of Figure 6 (b) lie closer to the buffer layer than do the bright luminescing areas of Figure 6 (a), i.e., the bright areas of 6 (b) imply the presence of a moat like feature surrounding this particular circular defect.

The large island surrounded by cracks observed on sample B73 (shown in the optical micrograph of Figure 2), provided some particularly dramatic CL images as illustrated in Figure 7. The CL images of Figure 7 were acquired at a beam energy of $7.5 \mathrm{keV}$ at a sample temperature of $>35 \mathrm{~K}$, under identical excitation and detection conditions except for the gain of the PMT amplifier which had to be reduced by a factor of 10 for Figure 7 (b). Again as for the CL images of Figure 6, we surmise that the bright acceptor luminescence emanates from near the buffer layer region of the sample, where in this case the luminescence is channeled up the cracks in the sample. The presence of the cracks thus allows us to simultaneously sample the luminescence properties of the surface of the epilayer and the region near to the buffer layer. We are effectively depth profiling the sample.

Figure 8 compares CL spectra obtained from the above cracked region of the sample and a smooth region of sample B73 respectively. Figure 8 complements the CL images of Figure 7 in illustrating how dramatically the luminescence properties are influenced by the depth at which the luminescence is sampled.

During our scanning electron and $C L$ studies of these samples we observed the secondary electron emission and $C L$ emission to increase under electron beam excitation. This phenomenon has been reported previously in CL imaging studies of GaN layers [11] and has been attributed to the electron beam causing contamination desorption [12], surface passivation [13] or annealing of the sample [14]. This phenomenon will be the subject of further work.

Finally, low temperature ( $35 \mathrm{~K}$ ) CL spectra were also acquired from sample B73 using the CL spectroscopy system, which allows continuous control of both the beam energy and the beam current. Figure 9 shows spectra acquired as a function of position moving down from the circular island surrounded by the cracks on B73. The spectra were acquired at a $10 \mathrm{keV}$, a $10 \mathrm{keV}$ electron beam has a Bethe range of $600 \mathrm{~nm}$. A number of interesting things are observed :

1. The spectra from the island itself are dominated by acceptor related luminescence.

2. The peak of the exciton band shifts to higher energy as we probe regions further away from the island. This can be attributed to a change in the strain. If we assume that the layer is under compressive strain due to the thermal mismatch between GaN and the sapphire substrate the observed blueshift implies an increase of the compressive strain as we move away from the island.

\section{Conclusions}

In conclusion, we have demonstrated that coupling imaging and luminescence techniques enables a more thorough characterisation of material, allowing the identification of the source of position dependent anomolous luminescence behaviour from samples. In this particular study, it was found that the morphological and optical quality of four samples 
with increasing buffer layer thickness (excepting that with the thinnest buffer layer of $30 \mathrm{~nm}$ ) are good, i.e., the films are predominantly smooth and the luminescence is dominated by excitonic luminescence. A number of circular islands are observed in all the films whose density decrease with increasing buffer layer thickness. A large island surrounded by cracks allowed the simultaneous investigation of luminescence emanating from both the surface of the epilayer and deeper into the epilayer revealing some interesting acceptor related luminescence.

\section{Acknowledgments}

We would like to thank David Clark, Ged Drinkwater and Jim Barrie for their technical support in constructing the cathodoluminescence systems.

\section{References}

[1] Shuji Nakamura, Gerhard Fasol, The Blue Laser Diode - GaN based Light Emitters and Lasers (Springer-Verlag, Heidelberg, 1997) .

[2] C. Trager-Cowan, K. P. O'Donnell , S. E. Hooper, C. T. Foxon , Appl. Phys. Lett. 68, 355-357 (1996).

[3] W. Van Der Stricht, I. Moerman, P. Demeester, J. A. Crawley, E. J. Thrush, P. G. Middleton, C. Trager-Cowan, K. P. O’Donnell, Mater. Res. Soc. Symp. Proc. 395, 231 (1996).

[4] C. Trager-Cowan, P. G. Middleton, K. P. O'Donnell, MRS Internet J. Nitride Semicond. Res. 1, 6 (1996).

[5] P. G. Middleton, C. Trager-Cowan, K. P. O’Donnell, T. S. Cheng, S. E. Hooper, C. T. Foxon, Mater. Sci. Eng. B 43 , 154 (1997).

[6] P. G Middleton, C. Trager-Cowan, A. Mohammed, K. P. O'Donnell, W Van der Stricht, I. Moerman, P. Demeester, unpublished (1997).

[7]A. Mohammed, C. Trager-Cowan, P. G. Middleton, K P O'Donnell, W. Van Der Stricht, I. Moerman, P. Demeester, "Hexagonal growth hillocks of MOCVD-grown GaN on (0001) sapphire" submitted to the Proceedings of the 10th Microscopy Of Semiconducting Materials, Oxford'97

[8] M Tchounkeu, O Briot, B Gil, JP Alexis, RL Aulombard, J. Appl. Phys. 80, 5352-5360 (1996).

[9] J. I. Pankove, Mater. Res. Soc. Symp. Proc. 162, 515 (1990).

[10] DA Turnbull, XLi, SQ Gu, EE Reuter, JJ Coleman, SG Bishop, J. Appl. Phys. 80, 4609-4614 (1996).

[11] K. Hiramatsu, H. Amano, I. Akasaki, J. Cryst. Growth 99, 375 (1990).

[12] S. Achour, A. Harabi, N. Tabet, Mater. Sci. Eng. B 42, 289 (1996).

[13] S. Myhajlenko, D. B. Davito, R. A. Puechner, J. L. Edwards, Phil. Mag. B 69, 553 (1994).

[14] B.G. Yacobi, D.B. Holt, Cathodoluminescence microscopy of inorganic solids (Plenum Press, New York, 1990) .

\section{Table 1}

Sample parameters

\begin{tabular}{|c||c||c|}
\hline Sample & Buffer & Epilayer \\
& $(\mathrm{nm})$ & $(\mathrm{mm})$ \\
\hline \hline B66 & 50 & 1.1 \\
\hline \hline B71 & 40 & 1.1 \\
\hline \hline B72 & 60 & 1.1 \\
\hline \hline B73 & 30 & 1.2 \\
\hline
\end{tabular}




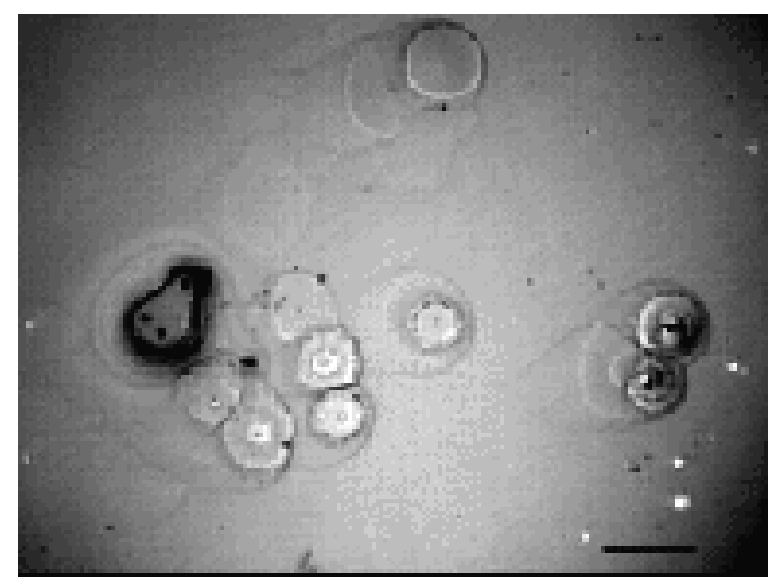

$a(673)$

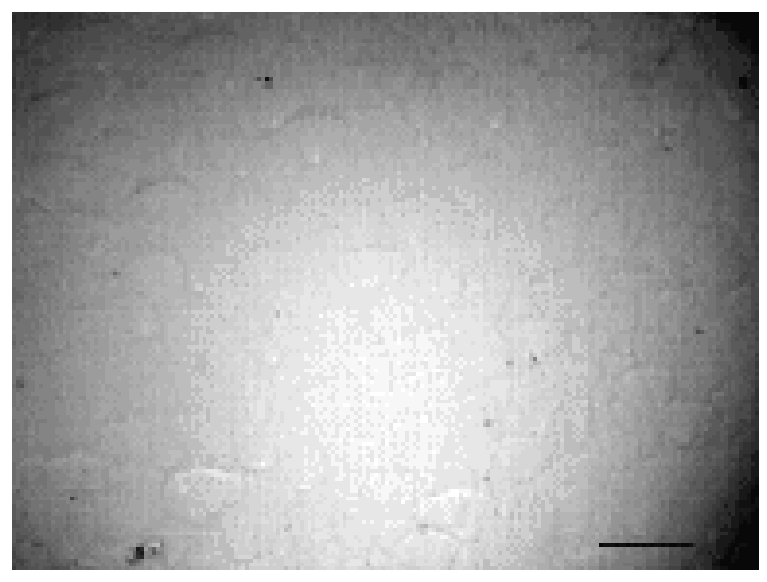

$c(\mathrm{~B} 60)$

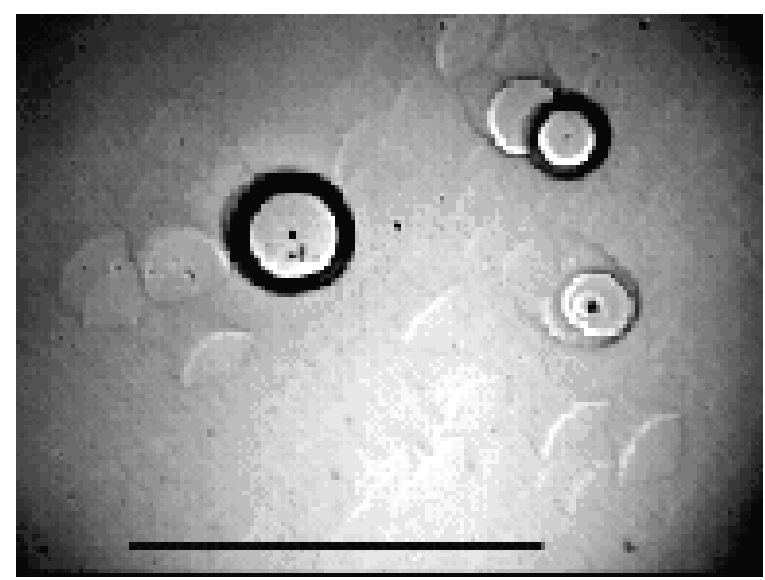

b $(B>1)$

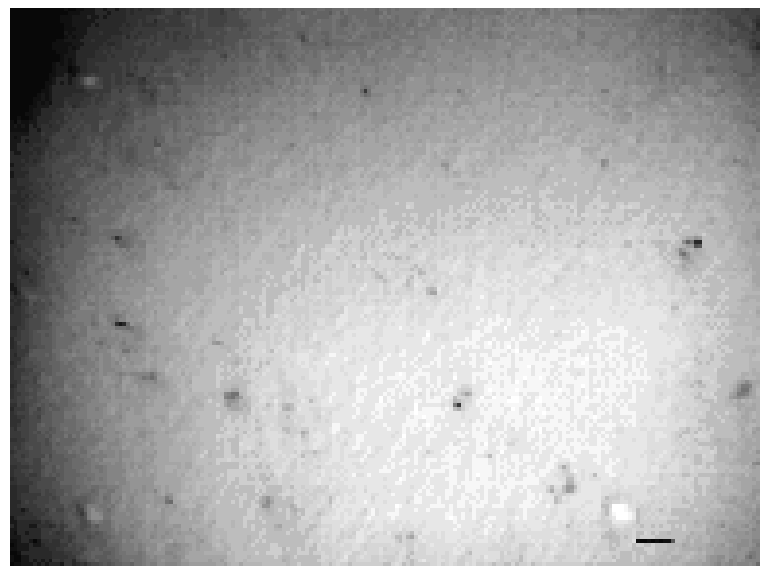

d (B72)

Figure 1. Optical micrographs from samples. The marker bar is $50 \mathrm{~mm}$ long in each case.

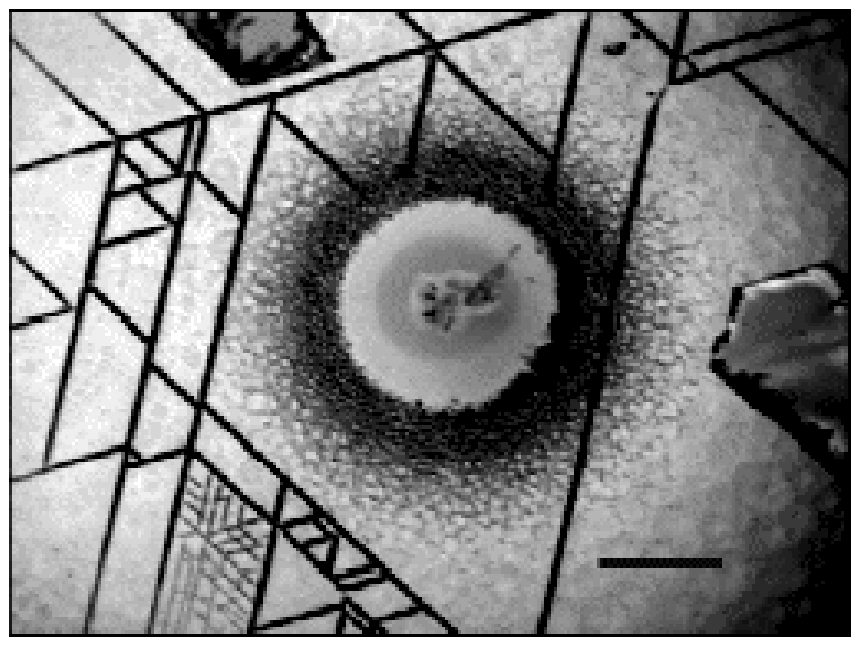

Figure 2. Image of a large island found on sample B73 which is surrounded by cracks in the $\langle 11 \overline{2} \quad 0>$ directions. The marker bar is $50 \mathrm{~mm}$ long. 

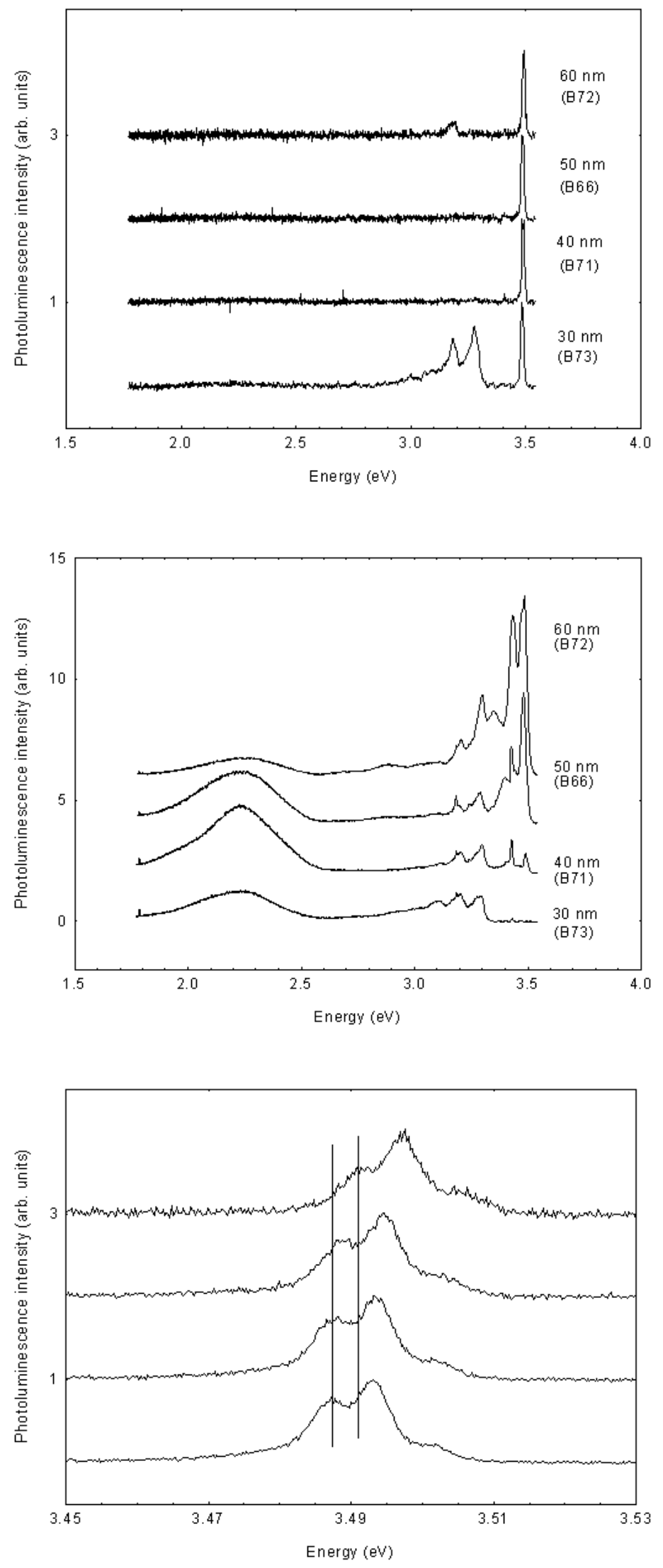

Figure 3. Low temperature PL spectra from the front face of all 4 samples.

Figure 4. Low temperature PL spectra from the backface (i.e., exciting through the substrate) for all 4 samples.

Figure 5. Low temperature PL spectra of the excitonic region as a function of position across sample B72. 


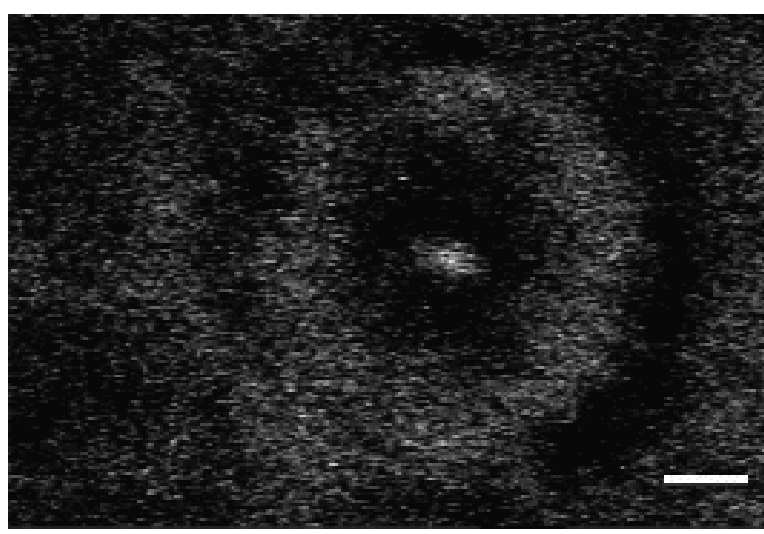

(a)

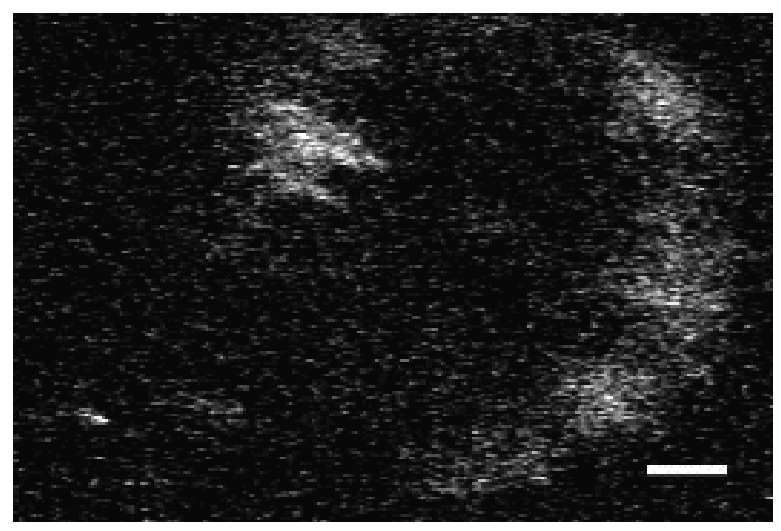

(b)

Figure 6. $\mathrm{CL}$ images acquired at (a) the exciton band at $350 \mathrm{~nm}(3.54 \mathrm{eV})$ and (b) the acceptor related band at 370 $\mathrm{nm}(3.35 \mathrm{eV})$. The marker bar (white lines) is $10 \mathrm{~m}$ long in each case.

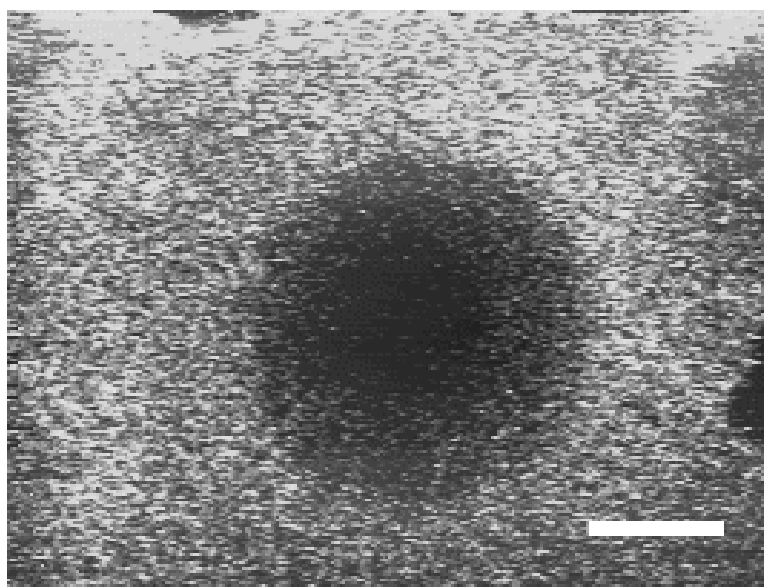

(a)

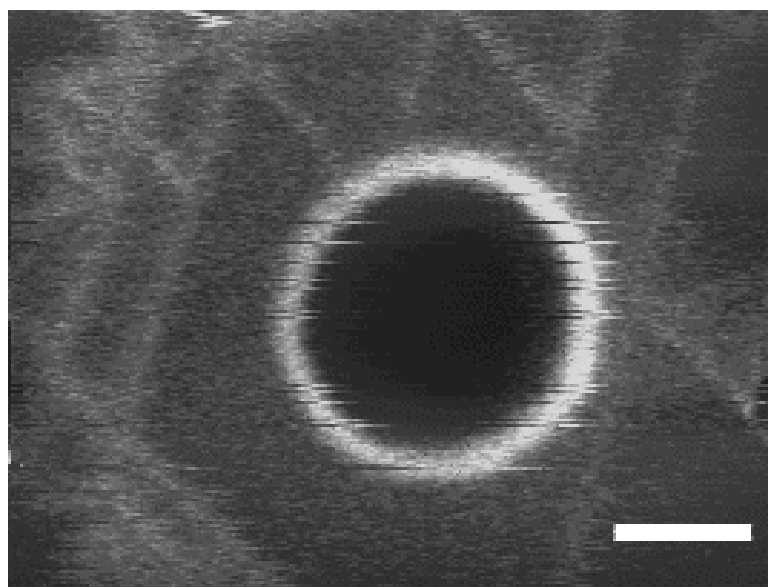

(b)

Figure 7. $\mathrm{CL}$ images acquired at (a) the peak of the exciton band at $356 \mathrm{~nm}(3.48 \mathrm{eV})$ and (b) at the peak of the acceptor related luminescence band at $392 \mathrm{~nm}(3.16 \mathrm{eV})$. The marker bar (white lines) is $50 \mathrm{~mm}$ long in each case.

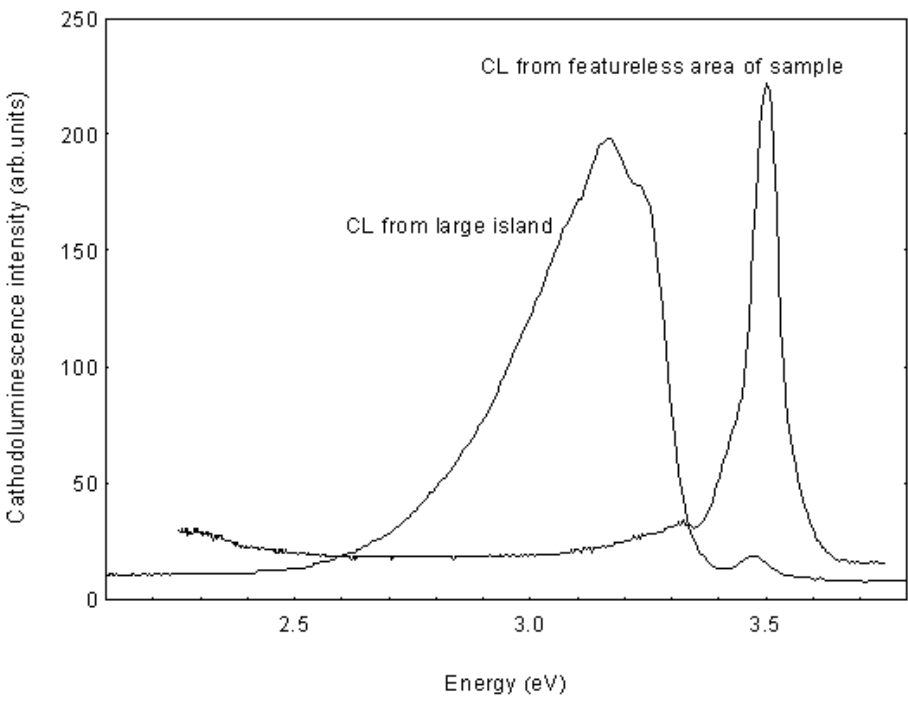

Figure 8. CL spectra from different regions of B73 (spectra not to scale). 

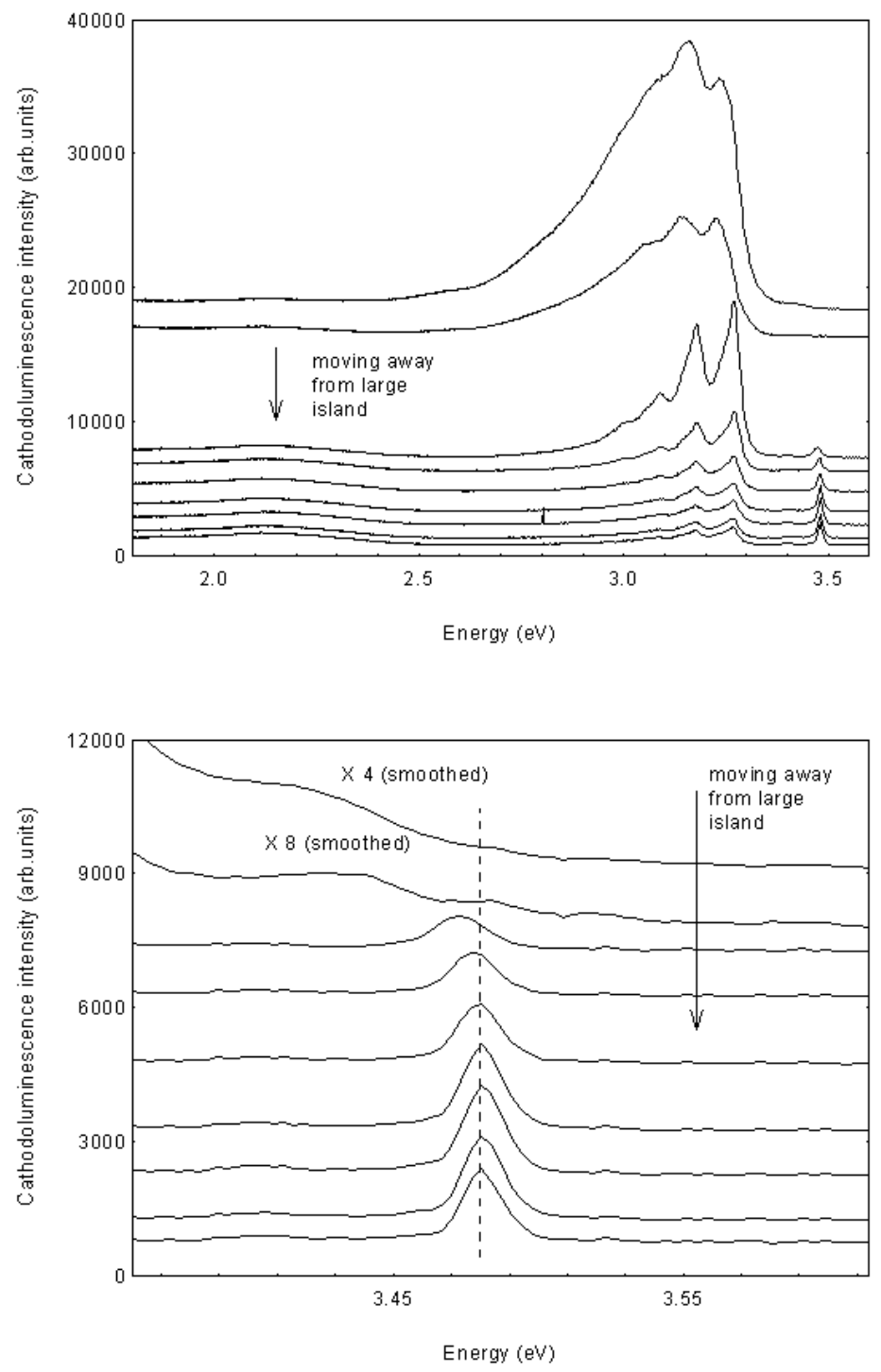

Figure 9a. Low temperature CL spectra acquired at as a function of position across sample B72.

Figure 9b. Low temperature CL spectra acquired at as a function of position across sample B73 for excitonic region of Figure 9a.

(C) 1997 The Materials Research Society 